\title{
Structure evolution and entropy increase in InBiGaSn equiatomic melt
}

\author{
R. Bilyk*, I. Shtablavyi, Y. Kulyk, S. Mudry \\ Physics of Metals Department, Ivan Franko National University of Lviv, Lviv, Ukraine
}

Received 27 March 2019, received in revised form 12 November 2019, accepted 13 December 2019

\begin{abstract}
Structure of quaternary InBiGaSn equiatomic molten alloy has been studied using the X-ray diffraction method at different temperatures within range from 375 up to $773 \mathrm{~K}$. Structure factors and pair correlation functions are analyzed and used to determine the main structure parameters. The structure of the quaternary melt is compared with one both for liquid constituent elements and partial binary melts. The results are interpreted with accounting for the thermodynamic data. It is shown that In-Bi interaction is the dominant factor, which promotes the formation of a structure with close to random atomic distribution.
\end{abstract}

Key words: low melting temperature alloys, cluster structure, high entropy alloys

\section{Introduction}

High entropy alloys (HEA) are a new class of metallic alloys whose entropy of mixing is increased due to a large number of constituent elements and formation of the solution with the random atomic distribution. Such alloys consist of 5 or more principal elements and the content of each element being between 5 and 35 at.\%. Presently, HEA are increasingly studied [1-6]. Structure and physical properties of these alloys are widely studied first of all by X-ray diffraction analysis, scanning electron microscopy, and measurement of mechanical characteristics. Therefore HEA are the multicomponent alloys with properties, significantly different from properties of elements, from which they are alloyed.

It is clear that it is not correct to consider each multi-component alloy as a HEA alloy. For many years the development of alloys for their application in the industry has been based on one or two principal elements, and other alloying components have been added in minor amounts. As a result, the principal elements have formed various phases with added elements (solid solutions, intermetallics), due to which the main properties have been improved. Later the wide group of alloys, known as composite materials, has been produced using metallic matrix and different kind impurities as a filler. Composites with an aluminum matrix belong to this type of materials, which are currently used in various areas of application.

Another kind of multi-component alloys that were developed during the last years of the previous century were amorphous metallic alloys. These alloys reveal the unique properties due to which they have a wide practical application but also consist of one or two principal elements. Amorphous alloys have a structure, which is, in many respects, similar to the structure of liquids, revealing the lack of long-range order. To produce such alloys, it is necessary to suppress the crystallization process, and it is commonly reached by rapid cooling.

Considering any multi-component alloy in the crystalline or amorphous state from a thermodynamic viewpoint, it should be noted that the formation of solid solutions phases or intermetallics, among other ones, results in the interatomic interaction and each of such phases can improve or impair the properties important for practical application. With the increasing of alloying elements amount, the probability of solid solutions formation also increases but some amount of intermetallics can also be formed. The fraction of structural units of each kind significantly affects most

*Corresponding author: e-mail address: roman.bilyk@lnu.edu.ua 
of properties. For instance, due to the presence of intermetallic phases, the strength of alloy can be increased with the dropping of plasticity. Intermetallic phases, as more ordered, lead to entropy decrease of alloy, whereas the solution phases increase this thermodynamic characteristic. The increase of entropy in solution phases leads to their higher thermodynamic stability, especially at elevated temperatures. It is important that in a liquid state, the Hume-Rosery rule about the atomic radius difference for constituent elements is not so strongly limited factor for atomic solution phase formation as in liquid state [7]. Therefore in the liquid state, such stability is higher in comparison with a solid one and it is possible to increase this stability in the solid state by quenching of liquid phase. For that reason at producing of HEA, it is important to choose the suitable constituent elements for multi-component alloy and create the thermodynamic and kinetic conditions necessary for the formation of atomic solution phases.

Due to the transition of multielement atomic solution from liquid to solid state without decomposition or ordering at quenching, HEA possess improved physical-chemical properties and first of all the mechanical ones. For that reason, the interest has grown recently in the study of $\mathrm{Al}, \mathrm{Fe}, \mathrm{Cu}$-based HEA as most widely used in various areas of practical application. Most of such alloys have a high melting temperature and need the protection from oxidation that is related to problems at studying, especially in a liquid state. It is easier to investigate the similar low melting temperature alloys, supposing that physical-chemical behavior at the formation of HEA is the same as for the alloys with high melting temperature. So, the low melting point of HEA makes them good model alloys for studying structure formation mechanism and interrelation between the entropy of mixing, atomic structure, and physical properties.

It is known that the main contribution to the entropy of mixing makes the configurational entropy, which equals:

$$
\Delta S_{\mathrm{conf}}=-k \ln w
$$

where $k$ is Boltzmann's constant, and $w$ is the number of ways in which the available energy can be mixed or shared among the particles of the system.

In the case of equiatomic alloy, this entropy per mole equals:

$$
\Delta S_{\mathrm{conf}}=R \ln N,
$$

where $R$ is a gas constant, and $N$ is a number of components.

For HEA, consisting of 5 elements, $\Delta S_{\text {conf equals }}$ $1.61 R$. Generally, it is accepted that HEA are alloys for which configurational entropy of mixing is greater than 1.5R. In our case of InGaSnBi alloy $(N=4)$, the configurational entropy of mixing equals $1.39 R$, and in this case, such alloy belongs to the group middle entropy alloy (MEA). Up today, the major attention of researches has been attracted to HEA and less to MEA. To make more understandable the mechanism of structure formation at alloying and to clarify the relationship between thermodynamic characteristics and structure in a liquid state it is suitable to consider the system with less number of principal elements but forming the alloy close to HEA. For that reason, this work aimed to study the structural state and thermodynamic properties of InBiGaSn liquid alloy of equiatomic concentration.

\section{Experimental}

The XRD measurements were carried out using a high-temperature X-ray diffractometer $\mathrm{CuK} \alpha$ -radiation, monochromatized with a LiF single crystal, installed into the primary beam, and Bragg-Brentano focusing geometry was used [8]. The temperature was measured with accuracy $\pm 2 \mathrm{~K}$. The scattered intensity was measured with accuracy higher than $2 \%$. Intensity curves were corrected on polarization and incoherent scattering [9-12]. After the normalization procedure, the structure factors have been calculated [13]:

$$
a(k)=\frac{I(k)}{N F^{2}(k)},
$$

where $a(k)$ is a structure factor, $N$ is a number of atoms, $F^{2}(k)$ is atomic scattering factor, $k=\frac{4 \pi \sin \theta}{\lambda}$ is wave-vector, $\lambda$ is the wavelength of X-rays, $\theta$ is scattering angle. Atomic distribution functions have been calculated from SF:

$$
4 \pi r^{2} \rho(r)=4 \pi r^{2} \rho_{0}+\frac{2 r}{\pi} \int_{0}^{\infty} k[a(k)-1] \sin (k r) \mathrm{d} k,
$$

where $r$ is a distance from any atom to another one, and $\rho_{0}$ is a mean atomic density. Also, pair correlation functions $g(r)$ have been calculated as:

$$
g(r)=1+\frac{1}{2 \pi^{2} r \rho_{0}} \int_{0}^{\infty} k[a(k)-1] \sin (k r) \mathrm{d} k .
$$

The melting-solidification process has been studied by the differential thermal analysis (DTA) using equipment interfaced with a computerized data system. The heating of samples was carried up to $500 \mathrm{~K}$ in an inert atmosphere with a linear heating mode and heating rate of 1.5 and $3 \mathrm{~K} \mathrm{~min}^{-1}$. 


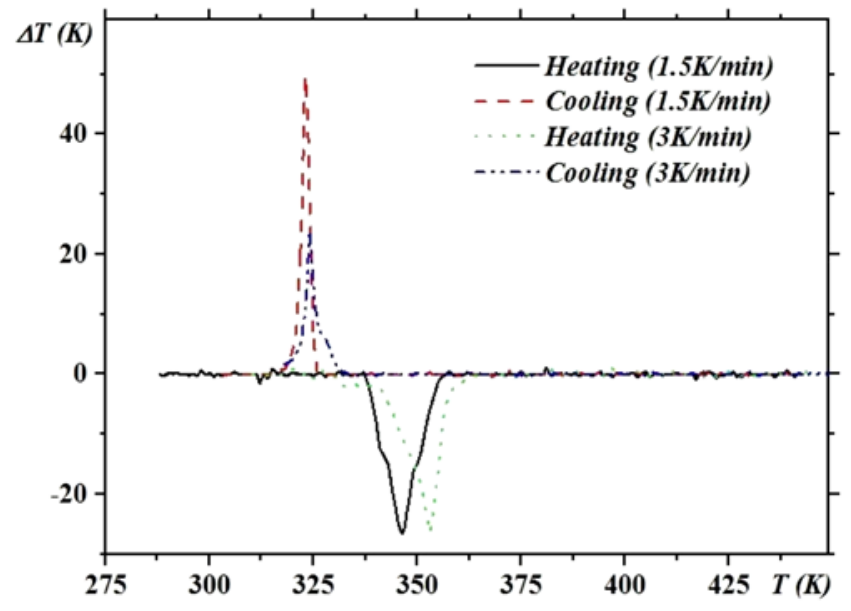

Fig. 1. DTA curves of InBiGaSn alloy.

\section{Results and discussion}

Currently, there is no reliable data on the phase diagrams of many four-component alloys. Therefore, the first issue that was to be clarified was the liquidus temperature for the InBiGaSn alloy. In this work, using the DTA method, it was established that at temperatures above $350 \mathrm{~K}$, the alloy is completely in a liquid state (Fig. 1). For this reason, the diffraction studies of the structure of this alloy were carried out above this temperature.

A more detailed analysis of Fig. 1 makes it possible to state the presence of premelting, which starts from $330 \mathrm{~K}$ (start-off temperature), and the onset temperature is measured to be $340 \mathrm{~K}$, which is usually called a melting point. However, as we can see, the temperature of complete melting is a bit higher. It is also seen that this melt shows the tendency to supercooling most probably due to the presence of $\mathrm{Ga}$ and $\mathrm{Sn}$ for which the supercooling is significant. In this case, the start-off temperature can mean initial melting which is more noticeable at the heating rate $3 \mathrm{~K} \mathrm{~min}^{-1}$. It should be noted that for the InBiGaSn alloy, no tail-off after the endothermic peak was found, as in the case of thermal analysis of similar alloys [14, 15].

X-ray diffraction studies of $\mathrm{InGaSnBi}$ of equi-atomic concentration have been carried out in liquid state at different temperatures and compared with the structure of InSn, GaBi, and InBi binaries as well as constituent elements.

Structure factors (SF) and pair correlation functions (PCF) have been analyzed and used to obtain the main structure parameters - distances to neighbor atoms, number of neighbor atoms, and size of clusters.

$\mathrm{SF}$ for equiatomic InGaSnBi alloy (Fig. 2) show the principal peak, which reveals the main features of constituent elements. First of all, there is a shoulder on the right-hand side of the principal peak, which is

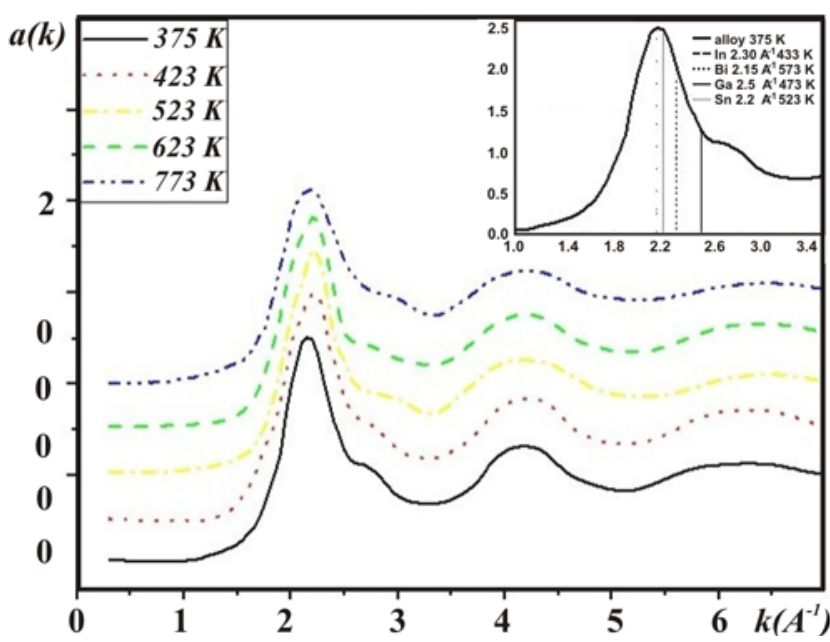

Fig. 2. SF of InBiGaSn liquid quaternary alloy at different temperatures.

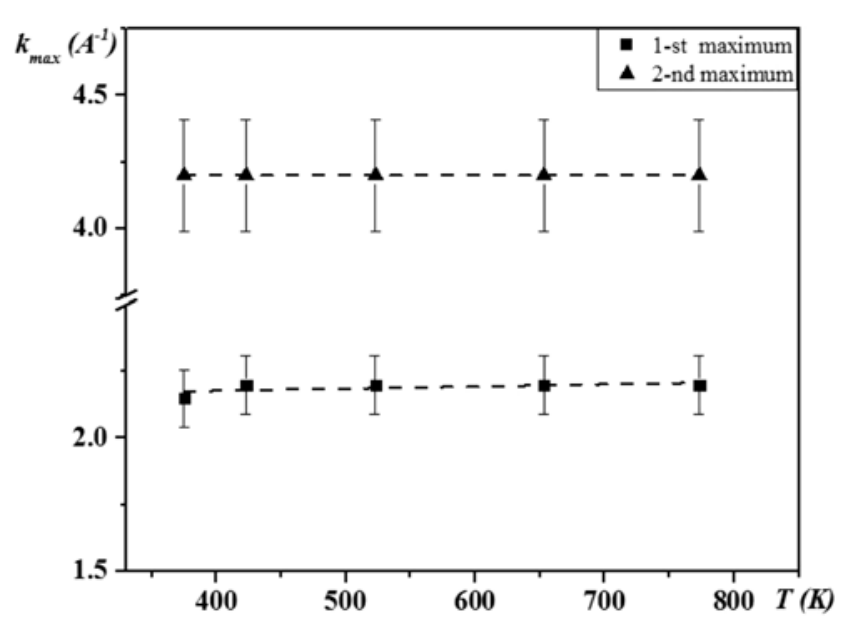

Fig. 3. Temperature dependence of SF peak positions.

also observed in SF for liquid $\mathrm{Ga}, \mathrm{Bi}$, and $\mathrm{Sn}$. But it is seen that this shoulder is less pronounced compared to similar shoulders in these three liquid metals. The position of this shoulder is very close to the position of one for liquid tin.

Principal peak position for the quaternary liquid alloy is rather close to one of the liquid tin than for other components of the alloy (Fig. 3).

With increasing the temperature up to $500 \mathrm{~K}$, the general features of SF are, in fact, unchangeable. Higher sensitivity to temperature is pronounced in the temperature dependence of principal peak height (Fig. 4). This parameter decreases due to the topologic disordering of atomic distribution at heating, which, according to the model of hard spheres, allows us to conclude that the density of atomic packing at heating decreases. It is clear that increasing the temperature 


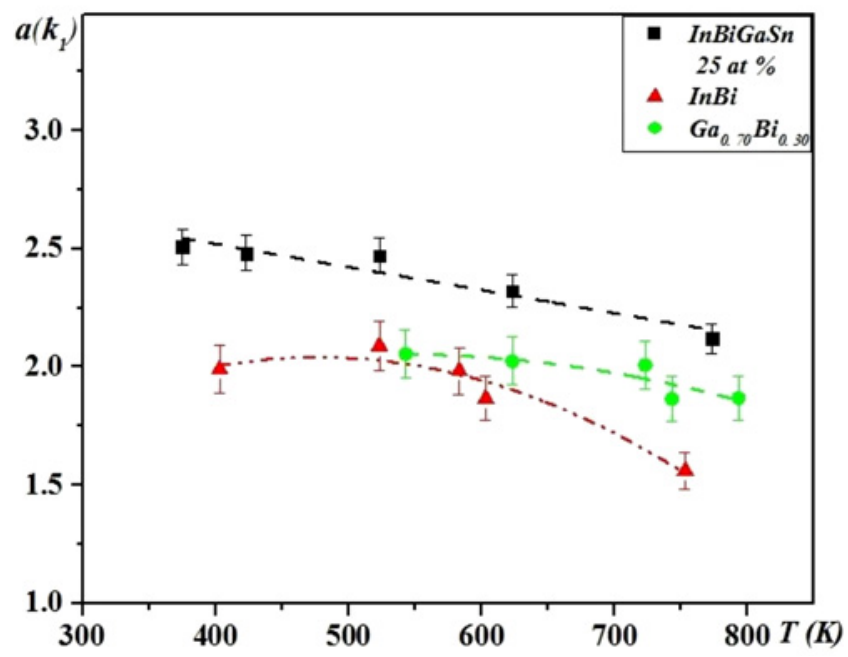

Fig. 4. Temperature dependence of SF principal peak height for InSnBiGa molten compared with principal peaks height for $\mathrm{InBi}$ and $\mathrm{Bi}_{0.30} \mathrm{Ga}_{0.70}$ alloys.

and decreasing the density makes better conditions for the mixing of atoms as a result of entropy increase.

For more accurate estimation of packing fraction in InBiGaSn liquid alloy, we used the PCFs, from which the atomic radii and the radii of first coordination spheres, as well as corresponding numbers of atoms, have been determined. Obtained data allowed us to calculate the fraction of volume, occupied by atoms:

$$
\eta=Z \frac{V_{\mathrm{at}}}{V_{\mathrm{sp}}} .
$$

Values of $V_{\text {at }}$ and $V_{\text {sp }}$ can be calculated using formulas:

$$
\begin{gathered}
V_{\mathrm{at}}=\pi R_{\max }^{3} / 6, \\
V_{\mathrm{sp}}=4 \pi R_{\min }^{3} / 3 .
\end{gathered}
$$

Consequently, the packing fraction coefficient equals:

$$
\eta=\frac{Z}{8}\left(\frac{r_{\max }}{r_{\min }}\right)^{3},
$$

where $Z$ is the area under the principal peak of radial distribution function (nearest neighbor atoms); $r_{\max }$ and $r_{\min }$ are first maximum and minimum positions in $\mathrm{PCF}$.

Obtained results (Fig. 5) show the decrease of packing density with the temperature that confirms the assumption pointed out above from the analysis of temperature dependence of principal peak height in SF.

Another structural parameter - the size of clusters, which was determined from the width of the principal

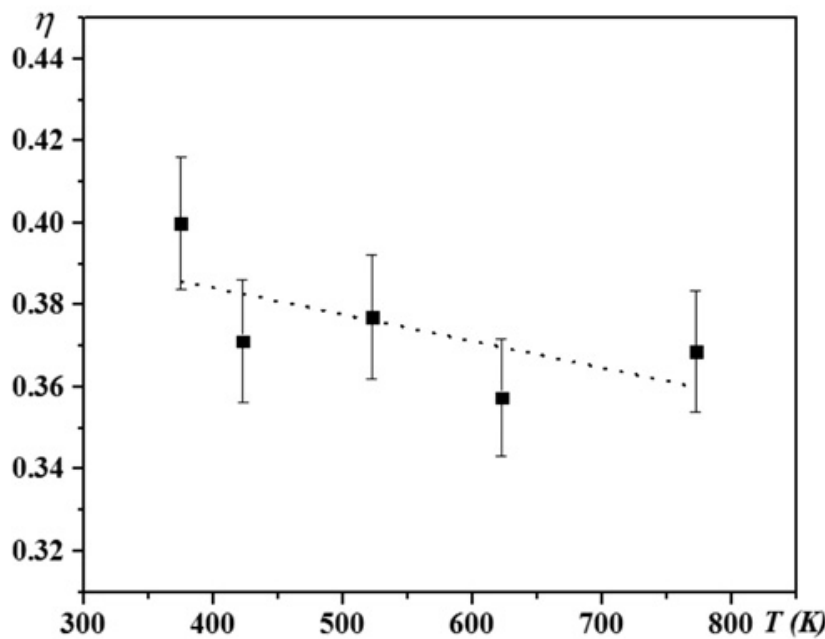

Fig. 5. Temperature dependence of packing density coefficient for InBiGaSn-molten alloy.

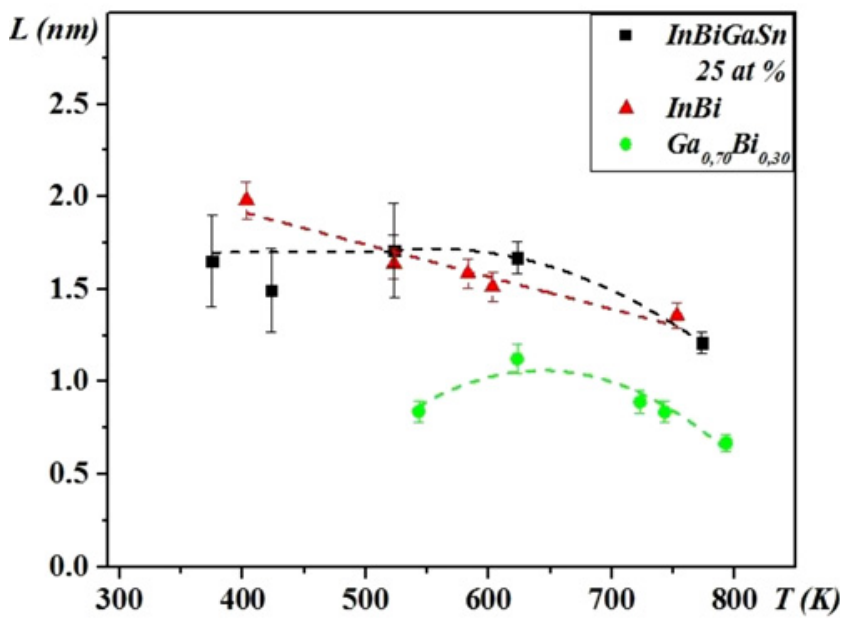

Fig. 6. Temperature dependence of cluster size liquid $\mathrm{In}_{0.25} \mathrm{Bi}_{0.25} \mathrm{Ga}_{0.25} \mathrm{Sn}_{0.25}$ alloy compared with cluster size for $\mathrm{InBi}$ and $\mathrm{Ga}_{0.70} \mathrm{Bi}_{0.30}$ melts.

peak in SF (Fig. 6), is unchangeable in the temperature interval of about $350-650 \mathrm{~K}$ and at further heating somewhat decreases.

Such local characteristic has the scale of short-range order and depends on temperature and concentration. As it is seen from Fig. 5, packing atomic density decreases with the temperature that is evidence of making more suitable conditions for mixing and as a result for entropy increasing. The reducing of cluster size also promotes the formation of a solution with a higher value of configurational entropy of mixing.

Another thermodynamic function, which is related to the structure of melts, is enthalpy of mixing. We have compared values of $\Delta H$ for constituent binary alloys with size factors (Fig. 7a) and with electroneg- 

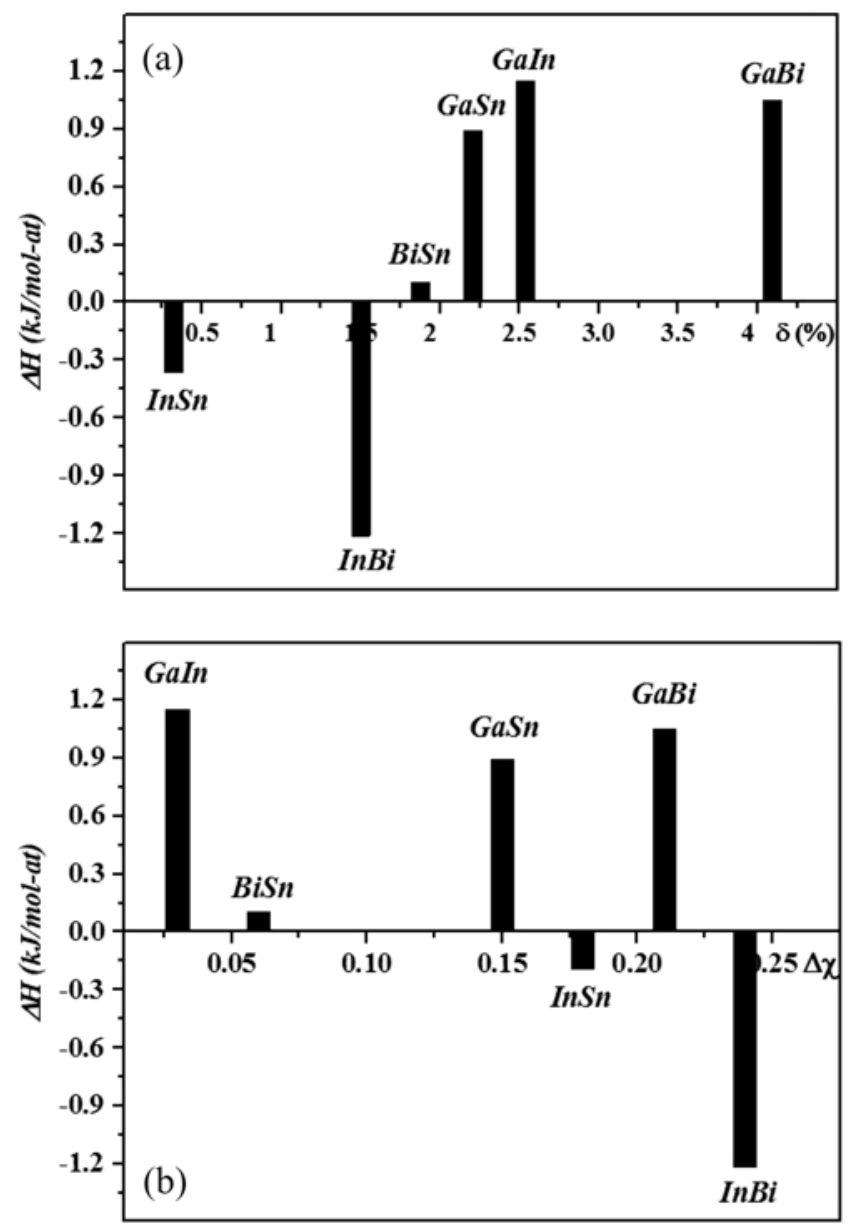

Fig. 7. Correlation between the enthalpy of mixing and size factor (a); correlation between the enthalpy of mixing and electronegativity difference (b).

ativity differences (Fig. 7b). As is seen, all three Ga-based binary alloys have maximum positive values of enthalpy of mixing, which corresponds to large values of size factors. Small positive $\Delta H$ is the characteristic of the Bi-Sn binary system. These systems show the phase diagrams with the miscibility gap in a liquid state $(\mathrm{GaBi})$ and eutectic diagrams (GaIn; BiSn; GaSn) and reveal the cluster structure with preferred interaction of like-kind atoms. Another two systems (InBi and InSn) show the negative values of $\Delta H$, and for that reason, their structure is supposed to be based on the preferred interaction of unlike kind atoms. Such a tendency is more pronounced for the InBi system, whose phase diagram reveals the existence of chemical compounds. In the case of this system, the size factor has a medium value, but the electronegativity difference is notable. Most probably, so large electronegativity difference is the main factor, which is responsible for the formation of chemical ordering in the atomic arrangement in a liquid state, and as a result, the entropy of mixing is reduced.

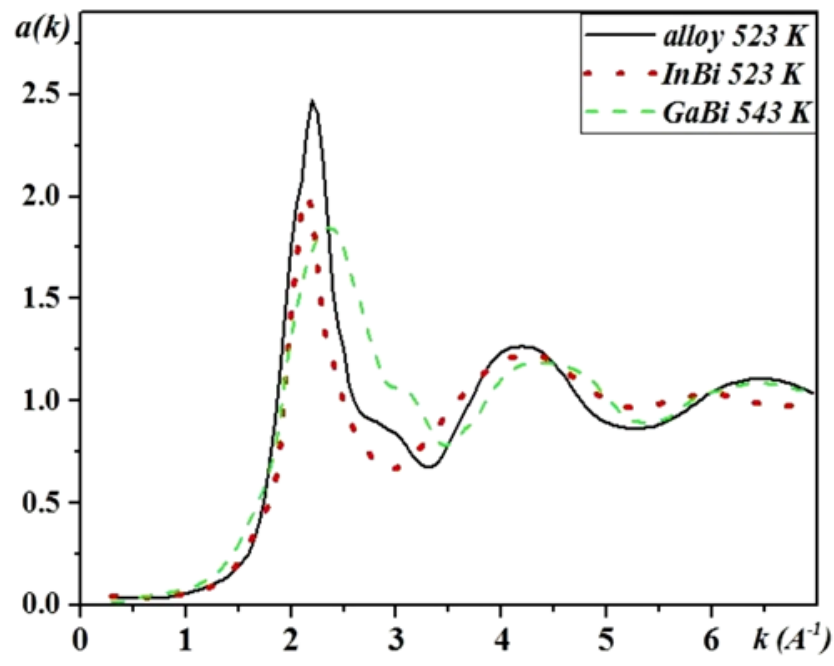

Fig. 8. SF for liquid $\operatorname{In}_{0.25} \mathrm{Bi}_{0.25} \mathrm{Ga}_{0.25} \mathrm{Sn}_{0.25}$ alloy compared with $\mathrm{SF}$ for InBi and $\mathrm{Ga}_{0.70} \mathrm{Bi}_{0.30}$ melts.

Thus, from the viewpoint of structural features, we have two different reasons, which decrease the entropy of mixing: large size factor, which promotes the formation of clusters with self-associated atoms, and large electronegativity difference, promoting the chemical ordering in atomic distribution. In the case of InGaSnBi equiatomic alloy, there are two subsystems ( $\mathrm{InBi}$ and $\mathrm{GaBi}$ ), which can be responsible for the most significant decrease of configurational entropy of mixing.

Alloys, corresponding to the rest of systems, which are of eutectic type, attempt to form the structure, whose atomic distribution is close to random atomic distribution and as a result, the entropy is increased. For that reason, we have compared the SF for multi-component melt with ones for InBi and $\mathrm{Ga}_{0.70} \mathrm{Bi}_{0.30}$ liquid alloys, which we obtained earlier [17-19] (Fig. 8).

As is seen, SF for quaternary InGaSnBi melt is more close to $\mathrm{SF}$ of liquid InBi intermetallic phase than for $\mathrm{Ga}_{0.70} \mathrm{Bi}_{0.30}$ melt. Particularly, principal peak profiles, including the shoulders, are, in fact, the same as well as the second maxima. Some discrepancies are observed in the height of the first and second peaks. Nevertheless, the structure of a multicomponent melt generally is close to the structure of $\mathrm{InBi}$ liquid alloy, which according to XRD data [17] and physical-chemical measurements, reveals the formation of chemically ordered atomic distribution. Therefore, obtained results allowed us to point out that the tendency to heterocoordinated atomic distribution in this binary melt is also repeated in quaternary InGaSnBi molten alloy. It can be supposed that the preferred interaction of unlike kind atoms is the dominant factor, determining the approach of atomic arrangement to random atomic distribution that leads 


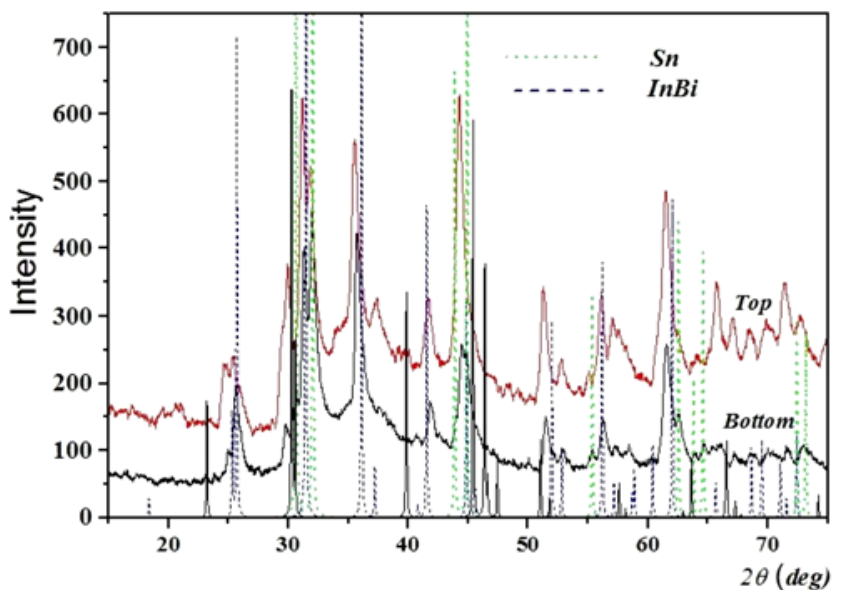

Fig. 9. Diffraction patterns for solid InBiGaSn equiatomic alloy.

Ta b le 1. Calculated values of configurational entropy

\begin{tabular}{lccc}
\hline Melt & $T(\mathrm{~K})$ & $S_{\text {conf }}^{\text {asym }} / k$ & $S_{\text {conf }}^{\text {sym }} / k$ \\
\hline $\mathrm{In}$ & 573 & 1.291 & 1.379 \\
$\mathrm{Bi}$ & 573 & 1.146 & 1.211 \\
$\mathrm{Ga}$ & 473 & 0.893 & 0.928 \\
$\mathrm{Sn}$ & 523 & 1.166 & 1.204 \\
$\mathrm{InBiGaSn}$ & 523 & 1.033 & 1.049 \\
\hline
\end{tabular}

to increasing of configurational entropy of mixing. We suppose that the atomic distribution of investigated four-component alloy is characterized by preferred interaction of $\mathrm{In}$ and $\mathrm{Bi}$-atoms with the $\mathrm{Ga}$ and $\mathrm{Sn}$ atoms which are diluted in chemically ordered $\mathrm{InBi}$ matrix. The formation of such a solution leads to the entropy of mixing increasing that in turn promotes the thermodynamic stability of solution resulting in the physical-chemical properties improving.

The phase analysis of solid alloys obtained upon rapid cooling (about $10^{3} \mathrm{~K} \mathrm{~s}^{-1}$ ) from the liquid state has also been studied using the XRD method. The identification of diffraction peaks (Fig. 9) shows that upon solidification, the formation of InBi intermetallic and tin is observed. Since the formation of InBi-compound requires an equal amount of indium and bismuth, it means that ternary eutectic alloy cannot be formed at all. It should be noted that the formation of InBi-compound is most probable from the viewpoint of thermodynamics because the enthalpy of mixing is less for it than for $\operatorname{In}_{2} \mathrm{Bi}$-compound [16].

However, some of the tin with all gallium can form a eutectic (melting point is $20.5^{\circ} \mathrm{C}$ ). Therefore, it is also seen the presence of tin in Fig. 9, and all gallium was in a liquid state as a part of the eutectic, which was liquid during the measurement at room temperature.
There are some discrepancies between diffraction patterns obtained from the top and bottom sides of the sample, but general characteristics are the same. Therefore, the preferred interaction of In and Bi atoms with InBi like chemical ordering in liquid InBiGaSn multicomponent alloy is inherited upon solidification.

Equation (9) was used to analyze the change in the entropy of the melt in the formation of a four-component alloy [20-23]:

$$
\frac{S_{\text {conf }}}{k}=-2 \pi \rho_{0} \int_{0}^{\infty} r^{2}\{g(r) \ln [g(r)]-[g(r)-1]\} \mathrm{d} r
$$

where $g(r)$ is pair correlation function, $\rho_{0}$ is mean atomic density, $r$ is a distance from any atom to another one, $k$ is Boltzmann's constant.

It is known that $S_{\text {conf }}$ is a measure of topologic ordering in the structure of liquid in comparison with the structure of ideal gas consisting of the same kind atoms. All liquids are the less simple metals, and, as was early mentioned, they show the asymmetric principal peak in SF and the shoulder on its right-hand side. To estimate what is an influence of such shoulder (indicating the presence of clusters, in which atoms are covalently bonded) on configurational entropy, we have calculated $S_{\text {conf }}$ using PCFs, obtained from SFs with real asymmetric principal peak $\left(S_{\text {conf }}^{\text {asym }}\right)$ and ones, obtained from symmetric such maximum $\left(S_{\text {conf }}^{\text {sym }}\right)$ (Table 1). Transformation of the first peak with a shoulder to symmetric one was carried out with the assumption that integral scattered intensity is unchangeable. As it is seen from the table, liquid In has a higher value of configurational entropy, whereas, for liquid Ga, it reveals minimum value. For $\mathrm{InSnGaBi}$ equiatomic melt $S_{\text {conf }}$ is less than for most constituent elements that allows concluding the increase of total entropy at the mixing of In, Bi, Ga, and Sn. There are also no significant changes in configurational entropy when clusters with covalent bonds are "removed" from liquid four-component alloy.

\section{Conclusions}

Equiatomic quaternary InBiGaSn molten alloy has a structure, which is another than the structure of each constituent element as well as for the system of the same atoms with the random atomic distribution. General features of such structure persist with heating, but some topologic disordering occurs. The structure of the quaternary melt is most close to the structure of InBi liquid intermetallic than for other binaries. Its structure is supposed to consist of an InBi-like heterocoordinated matrix in which atoms of $\mathrm{Ga}$ and $\mathrm{Sn}$ are diluted. The tendency to cluster formation both with chemical ordering (InBi) and self-associated 
ones ( $\mathrm{GaBi}, \mathrm{BiSn})$ is less pronounced in multicomponent melt comparing to binary ones that leads to the entropy of mixing increasing. Configurational entropy, calculated from diffraction data, shows the decrease at the formation of quaternary equiatomic mixture that is related to increasing of total entropy upon alloying.

\section{References}

[1] J. W. Yeh, S. K. Chen, S. J. Lin, J. Y. Gan, T. S. Chin, T. T. Shun, S. Y. Chang, Nanostructured high-entropy alloys with multiple principal elements: Novel alloy design concepts and outcomes, Advanced Engineering Materials 6 (2004) 299-303. doi:10.1002/adem.200300567

[2] M. A. Hemphill, T. Yuan, G. Y. Wang, J. W. Yeh, C. W. Tsai, A. Chuang, P. K. Liaw, Fatigue behavior of $\mathrm{Al}_{0.5} \mathrm{CoCrCuFeNi}$ high entropy alloys, Acta Materialia 60 (2012) 5723-5734.

doi:10.1016/j.actamat.2012.06.046

[3] Y. Zhang, T. T. Zuo, Z. Tang, M. C. Gao, K. A. Dahmen, P. K. Liaw, Z. P. Lu, Microstructures and properties of high-entropy alloys, Progress in Materials Science 61 (2014) 1-93.

doi:10.1016/j.pmatsci.2013.10.001

[4] B. Gludovatz, A. Hohenwarter, D. Catoor, E. H. Chang, E. P. George, R. O. Ritchie, A fracture-resistant high-entropy alloy for cryogenic applications, Science 345 (2014) 1153-1158. doi: $10.1126 /$ science.1254581

[5] Y. F. Ye, Q. Wang, J. Lu, C. T. Liu, Y. Yang, Design of high entropy alloys: A single-parameter thermodynamic rule, Scripta Materialia 104 (2015) 53-55. doi:10.1016/j.scriptamat.2015.03.023

[6] S. Q. Xia, X. Yang, T. F. Yang, S. Liu, Y. Zhang, Irradiation Resistance in $\mathrm{Al}_{x} \mathrm{CoCrFeNi}$ High Entropy Alloys, JOM 67 (2015) 2340-2344. doi:10.1007/s11837-015-1568-4

[7] W. Hume-Rothery, R. W. Smallman, C. W. Haworth, The Structure of metals and alloys, The Institute of Metals, London, 1969.

[8] A. F. Skryshevskyy, The structural analysis of liquids and amorphous solids, Nauka, Moscow, 1980.

[9] D. T. Cromer, J. T. Waber, Scattering factors computed from relativistic Dirac-Slater wave functions, Acta Crystallographica 18 (1965) 104-109. doi:10.1107/S0365110X6500018X

[10] K. Furukawa, The radial distribution curves of liquids by diffraction methods, Reports on Progress in Physics 25 (1962) 395-440. https://doi:10.1088/0034-4885/25/1/310
[11] Y. Waseda, The structure of non-crystalline materials, McGraw-Hill, New York, 1980.

[12] S. Mudry, I. Shtablavyi, U. Liudkevych, The relation between structure changes and thermal expansion in liquid indium, Physics and Chemistry of Liquids 55 (2017) 254-263. doi:10.1080/00319104.2016.1198482

[13] N. H. March, Liquid metals: Concept and theory, Cambridge University Press, Cambridge, 1990.

[14] C. H. Yang, S. Zhou, S. K. Lin, H. Nishikawa, A computational thermodynamics-assisted development of Sn-Bi-In-Ga quaternary alloys as low-temperature Pb-free solders materials, Materials 12 (2019) 631647. doi:10.3390/ma12040631

[15] S. W. Yoon, J. R. Soh, H. M. Lee, B. J. Lee, Thermodynamics-aided alloy design and evaluation of $\mathrm{Pb}$-free solder, SnBiInZn system, Acta Materialia 45 (1997) 951-960. doi:10.1016/S1359-6454(96)00253-4

[16] R. Hultgren, R. L. Orr, Ph. D. Anderson, K. K. Kelley, Selected values of thermodynamic properties of metals and alloys, John Wiley \& Sons, New York-London, 1963.

[17] I. Shtablavyi, S. Mudry, U. Liudkevych, Structure and thermal expansion mechanism of liquid InBi compound, Kovove Mater. 55 (2017) 351-356. doi:10.4149/km_2017_5_351

[18] S. Mudry, V. Sklyarchuk, A. Yakymovych, I. Shtablavyi, The structure and viscosity features in In-Bi neareutectic melts, Physics and Chemistry of Liquids 45 (2007) 675-681. doi:10.1080/00319100701434395

[19] S. Mudry, I. Shtablavyi, U. Liudkevych, Structure evolution and entropy changes of $\mathrm{Ga}_{0.7} \mathrm{Bi}_{0.3}$ liquid alloy, Physics and Chemistry of Liquids 57 (2019). doi:10.1080/00319104.2019.1594223

[20] H. J. Raveche, Entropy and molecular correlation functions in open systems. I. Derivation, The Journal of Chemical Physics 55 (1971) 2242-2250. doi:10.1063/1.1676399

[21] R. D. Mountain, H. J. Raveche, Entropy and molecular correlation functions in open systems. II Twoand three-body correlations, The Journal of Chemical Physics 55 (1971) 2250-2255. doi:10.1063/1.1676399

[22] A. Baranyai, D. J. Evans, Direct entropy calculation from computer simulation of liquids, Physical Review 40 (1989) 3817-3822. doi:10.1103/PhysRevA.40.3817

[23] N. Jakse, A. Pasturel, Excess entropy scaling law for diffusivity in liquid metals, Scientific Reports 6 (2016) 20689. doi: $10.1038 / \operatorname{srep} 20689$ 